\title{
Relations between hemispheric asymmetries of grey matter and auditory processing of spoken syllables in 281 healthy adults
}

\author{
Tulio Guadalupe $^{1} \cdot$ Xiang-Zhen Kong $^{1,2} \cdot$ Sophie E. A. Akkermans ${ }^{3,4} \cdot$ Simon E. Fisher ${ }^{1,3} \cdot$ Clyde Francks $^{1,3}$ (1)
}

Received: 12 August 2020 / Accepted: 14 January 2021

(c) The Author(s) 2021

\begin{abstract}
Most people have a right-ear advantage for the perception of spoken syllables, consistent with left hemisphere dominance for speech processing. However, there is considerable variation, with some people showing left-ear advantage. The extent to which this variation is reflected in brain structure remains unclear. We tested for relations between hemispheric asymmetries of auditory processing and of grey matter in 281 adults, using dichotic listening and voxel-based morphometry. This was the largest study of this issue to date. Per-voxel asymmetry indexes were derived for each participant following registration of brain magnetic resonance images to a template that was symmetrized. The asymmetry index derived from dichotic listening was related to grey matter asymmetry in clusters of voxels corresponding to the amygdala and cerebellum lobule VI. There was also a smaller, non-significant cluster in the posterior superior temporal gyrus, a region of auditory cortex. These findings contribute to the mapping of asymmetrical structure-function links in the human brain and suggest that subcortical structures should be investigated in relation to hemispheric dominance for speech processing, in addition to auditory cortex.
\end{abstract}

Keywords Dichotic listening $\cdot$ Voxel-based morphometry $\cdot$ Speech processing $\cdot$ Hemispheric language dominance $\cdot$ Brain asymmetry $\cdot$ Brain laterality

\section{Introduction}

Roughly $85 \%$ of people have left-hemisphere language dominance, as assessed with functional magnetic resonance imaging (fMRI) (Mazoyer et al. 2014), Wada testing (Keller

Tulio Guadalupe and Xiang-Zhen Kong contributed equally to the work.

Supplementary Information The online version contains supplementary material available at https://doi.org/10.1007/s0042 9-021-02220-z.

Clyde Francks

clyde.francks@mpi.nl

1 Language and Genetics Department, Max Planck Institute for Psycholinguistics, Wundtlaan 1, Nijmegen, The Netherlands

2 Department of Psychology and Behavioral Sciences, Zhejiang University, Hangzhou, China

3 Donders Institute for Brain, Cognition and Behaviour, Radboud University, Nijmegen, The Netherlands

4 Department of Human Genetics, Radboud University Medical Center, Nijmegen, The Netherlands et al. 2018), or functional transcranial Doppler sonography (Knecht et al. 2000). The exact population proportion showing left-hemisphere dominance varies across these methods and according to the different threshold criteria applied, as well as experimental details such as the language-related task to be performed (Mazoyer et al. 2014). In addition, most people show right-ear advantage when identifying spoken syllables in the dichotic listening paradigm, i.e. when presented with different syllables into the two ears (Hugdahl 2011). This reflects that the right ear is more directly connected to left hemisphere cortical auditory and speech processing regions than the left ear (and vice versa), and that these regions show leftward asymmetrical activation patterns in response to simple speech sounds (Hugdahl and Westerhausen 2016). Accordingly, the dichotic listening paradigm has become widely used as a relatively fast, easy and affordable way to index hemispheric dominance for speech processing (Westerhausen 2019). Under this paradigm, the population average extent of right-ear advantage depends on factors including stimulus order, the number of trials, topdown attentional aspects of the task, and the native language of participants (Westerhausen 2019; Bless et al. 2015b), as 
well as weak associations with sex and handedness (Bless et al. 2015a).

The structural basis of hemispheric language dominance remains unclear. At the population level, the human brain shows various left-right asymmetries of macrostructure, including hemispheric differences of cortical surface area and thickness in inferior frontal and lateral temporal regions, which are important for language and/or auditory processing (Kong et al. 2018; Toga and Thompson 2003; Geschwind and Levitsky 1968). Microstructural asymmetries in auditory and language-related cortical regions have also been reported based on post mortem histology (Hutsler and Galuske 2003; Hutsler 2003; Chance 2014) or neurite orientation and dispersion density imaging (Ocklenburg et al. 2018; Schmitz et al. 2019), including left-right differences in dendrite and axon density. Asymmetries of regional anatomy around the Sylvian fissure (i.e. the fissure that separates the frontal and parietal lobes from the temporal lobe) have a developmental origin in utero (Kasprian et al. 2011) and are, therefore, likely to arise from a genetically regulated program that favours hemispheric differences (Francks 2015; de Kovel et al. 2017, 2018; Ocklenburg et al. 2017).

However, peri-Sylvian regions also show a high degree of variability between people in terms of sulcal and gyral anatomy and asymmetry (Tzourio-Mazoyer et al. 2018). There is also considerable variation in functional laterality for language, with around $10-15 \%$ of people having a bilateral pattern without clear dominance, and roughly $1 \%$ having completely reversed, right-hemisphere language dominance (Mazoyer et al. 2014). In recent years it has become clear that the population variances of language-related structural and functional asymmetry are only weakly correlated (Tzourio-Mazoyer et al. 2018; Zago et al. 2017; Josse et al. 2009; Keller et al. 2018; Ocklenburg et al. 2014; Tzourio-Mazoyer and Seghier 2016; Greve et al. 2013; Jansen et al. 2010), which raises the question of how tightly asymmetrical structure is related to asymmetrical function. For example, it is uncertain whether surface area asymmetries of superior temporal auditory cortex correlate with hemispheric language dominance (Greve et al. 2013), or only with variation in more restricted regional functional asymmetries during auditory language tasks (Tzourio-Mazoyer et al. 2018). Regardless, these studies found only subtle structure-function associations, not involving substantially predictive relationships.

We have previously suggested (Kong et al.) that asymmetrical structure may indeed be coupled to function in the population-average form that characterises the majority of people, but when asymmetrical development of the embryonic brain is perturbed in a minority of people, different aspects of asymmetrical organization may become dissociated from each other as re-organization occurs.
This would be consistent with weak correlations between measures of asymmetrical structure and function in the adult population. In this context, it is still informative to identify subtle associations between population variances in asymmetrical structure and function, as the anatomical regions implicated may be especially important for underlying functional asymmetry in most people. Random variation in the early embryo may be the most important source of individual differences in adult brain asymmetry, because despite the emergence of population-average brain asymmetries in utero, variation in brain and behavioural asymmetries tends to be only weakly heritable (Kong et al. 2018; Eyler et al. 2013; de Kovel et al. 2019a, b; Postema et al. 2020; de Kovel and Francks 2019; McManus et al. 2013), and only slightly affected by early life factors such as birthweight, twinning or breastfeeding (de Kovel et al. 2019a, b; Postema et al. 2020; de Kovel and Francks 2019; McManus et al. 2013).

As regards structural correlates of functional asymmetry measured by dichotic listening specifically, only a small number of studies have been performed. In a study of 29 healthy adults (Ocklenburg et al. 2014), variation in nerve fibre tracts that link core language-related cortical regions was linked to the extent of right-ear advantage in the dichotic listening task. Specifically, tract volume and fractional anisotropy of the left arcuate fasciculus, and volume of the right uncinate fasciculus, were positively associated with the strength of functional lateralization. Another study of 24 people with schizophrenia, and 25 controls, reported that better performance for the left-ear stimulus was associated with larger grey matter volume in the left frontal lobe, for the two groups combined (Nygard et al. 2013). A third study found that among 39 people with early onset schizophrenia, those with an absence of right-ear advantage had a reduction of left temporal lobe volume compared with those with normal right-ear advantage, and also compared to a set of matched controls (Collinson et al. 2009). While these findings are intriguing, the relatively low sample sizes warrant caution, as statistical power would have been low to detect subtle links between structure and function. Low statistical power in a study not only reduces the chance of detecting true effects, but also the likelihood that statistically significant results reflect true effects (Munafo and Flint 2010).

Here we performed the largest study to date aimed at finding relations between hemispheric asymmetries of auditory syllabic processing and of grey matter, based on MRI and dichotic listening data from 281 healthy adults. We took a brain-wide mapping approach, given that previous studies have not reliably limited the search space, and that hemispheric asymmetries of auditory processing may 
relate to broader network-level functional asymmetries ( $\mathrm{Ji}$ et al. 2019). However, we also considered superior temporal regions of primary and secondary auditory cortex (Moerel et al. 2014) as a specific candidate set for focused analysis.

\section{Methods}

\section{Participants}

The BIG study was initiated in 2007 and comprises healthy volunteers, including many university students, who have participated in studies at the Donders Centre for Cognitive Neuroimaging, Nijmegen, The Netherlands (Franke et al. 2010). Participants underwent anatomical (T1-weighted) MRI scans as part of their involvement in diverse smaller-scale studies at the Donders Centre. All participants gave written, informed consent to participate in BIG. After inclusion of their scan in BIG, participants were re-contacted several times over the course of the subsequent years, to request that they complete online test and questionnaire batteries, one of which included the dichotic listening task (below). Ethical approval for the BIG study and its extensions was from the 'Commissie Mensgebonden Onderzoek' (Committee on Research Involving Human Subjects) of the region Arnhem-Nijmegen.

At the time of the current study, 643 participants had completed the relevant battery. After matching the available dichotic listening and neuroimaging data, and applying quality control criteria to both (specified below), in total 281 participants (180 females) were used in the dichotic-neuroimaging association analyses in this study. The mean age at MRI scanning for these 281 participants was 25.7 years old, SD 10.6 years, range from 18.1 to 69.0 years. The time that had passed between MRI scanning and dichotic listening ranged from 1.6 months to 15.3 years, mean 5.8 years (SD 3.7 years) (some scans were retrospectively included in BIG from as far back as 2003). The mean age was 31.4 years at the time of completing the online test battery, SD 11.1 years, range from 18.8 to 74.4 years.

Handedness was assessed with a questionnaire (a choice of strongly right-handed/moderately right-handed/ambidextrous/moderately left-handed/strongly left-handed, in Dutch, from which the 'moderate' data were then pooled together with the 'strong' data for each hand). Of the 281 participants with dichotic listening and MRI data, there were 10 left-handers, 1 who indicated mixed hand preference, and 9 with missing handedness data. This rate of left-handedness $(3.7 \%)$ is lower than the general population rate of roughly 10\% (de Kovel et al. 2019b; McManus 2009; Papadatou-Pastou et al. 2020), as left-handedness was an exclusion criterion in some of the separate studies that contributed to the BIG dataset.

\section{Neuroimaging}

Scanning was conducted using either a $1.5 \mathrm{~T}$ Siemens Avanto or Sonata scanner, or a 3.0 T Siemens TIM Trio, Skyra, Prisma, or Prisma-Fit scanner (see Table 1 for the numbers of participants by scanner). A variety of different scanning protocols was used (Supplementary Table 1). T1-weighted images were available for 293 participants who had post-quality-control dichotic listening laterality indexes (below). Twelve participants were excluded because of imaging quality: six during an initial quality control procedure to identify visibly large artefacts (e.g. caused by head motion) which would likely prevent brain structure from being properly delineated, five because of skull stripping failure, and one with a technical failure related to file reading. Brain structural data was analysed with FSL-VBM (Douaud et al. 2007), http://fsl.fmrib.ox. ac.uk/fsl/fslwiki/FSLVBM), an optimised VBM protocol (Good et al. 2001) carried out with FSL (v5.0) tools (Smith et al. 2004). First, structural images were brainextracted and grey matter-segmented before being registered to the MNI 152 standard space using non-linear registration (Andersson et al. 2010). The resulting images were averaged and flipped along the $x$-axis to create a left-right symmetric, study-specific, grey matter template. Second, participants' grey matter images were non-linearly registered to this study-specific template and "modulated" to correct for local expansion or contraction due to the non-linear component of the spatial transformation. The modulated grey matter images were then smoothed with an isotropic Gaussian kernel with a sigma of $2.55 \mathrm{~mm}$ (fullwidth at half-maximum $=6 \mathrm{~mm}$ ). Next, voxel-wise asymmetry index $(\mathrm{AI})$ maps were calculated using the formula $(L-R) /((L+R) / 2)$ for each pair of left-right corresponding voxels, per participant.

\section{Dichotic listening}

The dichotic listening task was performed at home via web-browser, implemented by the Delosis company

Table 1 The numbers of participants per MRI scanner

\begin{tabular}{lll}
\hline Scanner & Field strength & Participants \\
\hline Siemens Avanto & $1.5 \mathrm{~T}$ & 66 \\
Siemens Sonata & $1.5 \mathrm{~T}$ & 14 \\
Siemens TIM Trio & $3 \mathrm{~T}$ & 109 \\
Siemens Skyra & $3 \mathrm{~T}$ & 46 \\
Siemens Prisma & $3 \mathrm{~T}$ & 30 \\
Siemens Prisma-Fit & $3 \mathrm{~T}$ & 16 \\
Total & - & 281 \\
\hline
\end{tabular}

See also Supplementary Table 1 for scanning protocols 
(Delosis.com). Participants used either their own earphones, or earphones provided to them by mail. Participants were first presented with the test instructions and four questions dealing with the correct left-right placement of their headphones. For three of the questions, participants selected one of three possible options for the perceived location of a test tone (left ear, right ear or both). The fourth question was answered by moving a slider on a scale ranging from 0 (left) to 100 (right) for a tone presented equally to both ears. 410 participants correctly answered all four of the four setup questions (for the slider scale, responses between 30 and 70 were accepted as correct). Data from participants who had answered one or more of the four questions incorrectly were discarded. Participants were also presented with an explicit instruction that the "ga" syllable referred to the /g/ sound as used in common loanwords into the Dutch language, rather than the Dutch pronunciation $(/ x /$ or $/ \gamma /$ which is not a stop-consonant)

The dichotic listening task involved six consonant-vowel syllables as stimuli. This format has been used previously in a functional imaging study (Hugdahl and Westerhausen 2016), and was also shown to be effective in a smartphone application outside a laboratory environment (Bless et al. 2013, 2015b). Nonetheless, our exact implementation involved new recorded stimuli that were suitable for Dutch first-language participants, and for integration into our software application.

The stimuli consisted of consonant-vowel (CV) syllables using the six stop-consonants: /ba/, /da/, /ga/, /ta/, /ka/ and / pa/. The syllables were spoken with constant intonation and intensity by a male native Dutch speaker, 32 years old. These syllables differ in their voicing and can be grouped in two categories. Voiced stop-consonant syllables have a short (S) voice of onset time (VOT), while voiceless stop-consonant syllables have a long (L) VOT. The voiced stop-consonant syllables (S), /ba/, /da/ and /ga/, had durations of 450, 467 and $506 \mathrm{~ms}$ respectively. The voiceless stop-consonant syllables (L), /ta/, /ka/ and /pa/, had durations of 305, 300 and $310 \mathrm{~ms}$, respectively. The syllables were normalized for loudness and each pair was temporally aligned for simultaneous release of the stop-consonants, using Audacity, v2.1 (https://audacityteam.org/). All syllable combinations were formed in both left-right and right-left orientations, resulting in a total of 36 pairs, i.e. 30 dichotic and 6 homonym pairs (Table 2).

Each of the syllable pairs was presented once. After each presentation, participants clicked on the button corresponding to the syllable they could best identify, from a choice of all six. A new pair was presented automatically after $4000 \mathrm{~ms}$. Correct responses were recorded when a syllable was correctly identified for either ear, and missing responses were considered incorrect. Participants were
Table 2 The 30 dichotic stimulus pairs, arranged according to voice onset time (VOT) categories

\begin{tabular}{llll}
\hline LL & SS & LS & SL \\
\hline ka_pa & ba_da & ka_ba & ba_ka \\
ka_ta & ba_ga & ka_da & ba_pa \\
pa_ka & da_ba & ka_ga & ba_ta \\
pa_ta & da_ga & pa_ba & da_ka \\
ta_ka & ga_ba & pa_da & da_pa \\
ta_pa & ga_da & pa_ga & da_ta \\
& & ta_ba & ga_ka \\
& & ta_da & ga_pa \\
& & ta_ga & ga_ta \\
\hline
\end{tabular}

$L L$ long to both ears, $S S$ short to both ears, $L S$ long to left, short to right, $S L$ short to left, long to right

excluded if they had an error rate $>80 \%$ for dichotic trials, or an error rate $>50 \%$ for homonym trials, following a previously reported protocol (Bless et al. 2013, 2015b).

We computed average correct responses for the four different presentation categories: (1) when both ears were presented long-VOT syllables at once, (2) when both ears were presented short-VOT syllables, (3) when a long-VOT syllable was presented to the left and a short-VOT syllable to the right, and (4) when a short-VOT syllable was presented to the left and a long-VOT to the right (Table 2). We noticed that for syllable pairs of unequal voicing categories (i.e. LS and SL) correct responses were given overwhelmingly for the short VOT syllable, regardless of ear presentation (Fig. 1; see the Discussion for an interpretation of this issue).

We therefore computed the dichotic listening laterality index based only on presentations where both syllable pairs were of equal voicing group (i.e. LL and SS; Table 2). Laterality indices were calculated for each subject as the proportional difference in correct responses between right and left presented syllables: $\mathrm{LI}=$ (\# correct Right - \# correct Left) / (\# correct Total), with positive scores denoting a right-ear advantage for identifying syllables.

\section{Voxelwise general linear model analysis}

General linear modelling (GLM) was applied to examine the association between grey matter AI maps (voxel-wise AIs as dependent variables) and the dichotic listening laterality index (independent variable). Confound variables in the GLM were age at MRI scanning (continuous), years elapsed between scanning and dichotic listening (continuous), sex (binary), and scanner (categorical, as dummy coding, which also accounted for field strength differences; Table 1). Multiple comparison correction across the whole brain was performed on statistical maps using the easythresh program (voxel-level $Z>3.09$, and cluster-level $p<0.05$ ) implemented within FSL. Clusters 
Fig. 1 Mean correct responses for the four different presentation categories by voice onset time (VOT) category. $L L$ long to both ears, $S S$ short to both ears, $L S$ long to left, short to right, $S L$ short to left, long to right. 95\% confidence intervals (plus and minus two standard errors) are shown on each bar
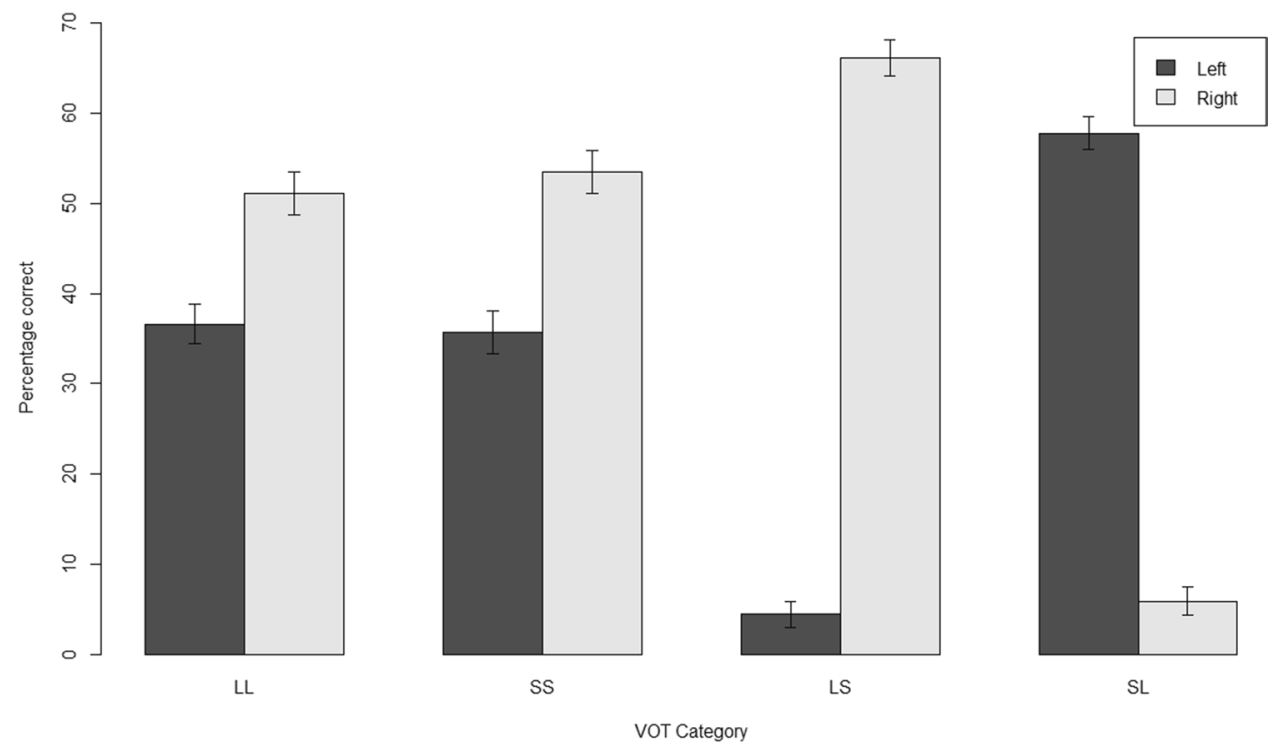

$\mathrm{SL}$ were given post hoc anatomical labels according to the Harvard-Oxford atlases of cortical and subcortical brain regions (Goldstein et al. 1999; Frazier et al. 2005) and the cerebellar atlas from FSL (Diedrichsen et al. 2009).

In addition, we used a relatively loose threshold (voxellevel $Z>2.58$ \& cluster-level $p<0.05$ ) to examine three neighbouring candidate regions of interest within our symmetric, study-specific grey matter template, using a voxelwise probability threshold of $50 \%$ for belonging to a given region (according to left-hemisphere regional definitions within the Harvard-Oxford cortical atlas (Goldstein et al. 1999)). The regions were, from anterior to posterior along the superior temporal lobe: 'Heschl's gyrus', 'Superior Temporal Gyrus, posterior division', and 'Planum temporale'. The intention was to detect any potential trends of association that might indicate structure-function relations within auditory cortex, but that were too weak to detect using standard correction for voxel-wise multiple testing. However, Bonferroni correction was applied for multiple testing across the three regions (i.e., $0.05 / 3=0.017$ ).

We did not include handedness in the modelling, as only 10 participants were left-handed (see above) and there was no association of handedness with the dichotic listening laterality index (Left vs. Right: $t=-0.93, p=0.36$; see also Discussion). However, we did repeat the analyses after excluding these 10 individuals, as a sensitivity analysis.

To further investigate each hemisphere's contribution to associations identified through the voxel-wise AI analysis, we also performed post hoc analyses of the associations between the dichotic listening laterality index and the grey matter volumes at the homologous locations in the two hemispheres separately, for the peak coordinates per cluster, using Pearson correlation.

\section{Results}

The mean dichotic listening laterality index in the 281 participants with dichotic listening and MRI data was 19.6 $(\mathrm{SD}=35.1)$, which indicates a higher percentage of correct responses for syllables presented to the right ear, i.e. the expected right-ear advantage at the population level (Fig. 2). Only $23 \%$ of individuals had negative indexes, which indicate left-ear advantage, i.e. 64 individuals. Another 11\% (31 individuals) had index values of zero, indicating no lateralized ear advantage.

In brain-wide analysis, the dichotic listening laterality index was significantly and positively associated with regional grey matter asymmetry in the amygdala (cluster size $=153$ voxels, $p=0.0073$; peak voxel $Z=3.98$ at MNI coordinate $-14,-2,-20$ ) and lobule VI of the cerebellum (cluster size $=134$ voxels, $p=0.013$; peak voxel $Z=3.85$ at MNI coordinate $-26,-66,-18$ ) (Fig. 3a). More details of the models at the peak voxels are in Supplementary Table 2. Post hoc correlation analysis of unilateral grey matter volumes at the peak coordinates (i.e. in each hemisphere separately) with the dichotic listening laterality index showed that effects at both clusters involved greater right sided volumes in participants with increasingly atypical left-ear advantage (Fig. 3c)(Amygdala: left $r=0.02, p=0.697$; right $r=-0.14, p=0.0227$. Cerebellum VI: left $r=0.01$, $p=0.840$; right $r=-0.12, p=0.0524$ ).

When focusing on three neighbouring regions of interest from the auditory cortex, using a relatively loose voxellevel $Z$ threshold to detect any tentative trends of association (Methods), there was a small cluster on the posterior superior temporal gyrus (pSTG; cluster size $=3$ voxels, corrected $p=0.014$; peak voxel $Z=2.78$ at MNI coordinate $-62,-30,4$; Fig. 3b), within which grey matter asymmetry 
Fig. 2 Frequency distribution of the dichotic listening laterality index. Negative values indicate left-ear advantage, positive values indicate right-ear advantage. Right-handers and left-handers are indicated by different shading

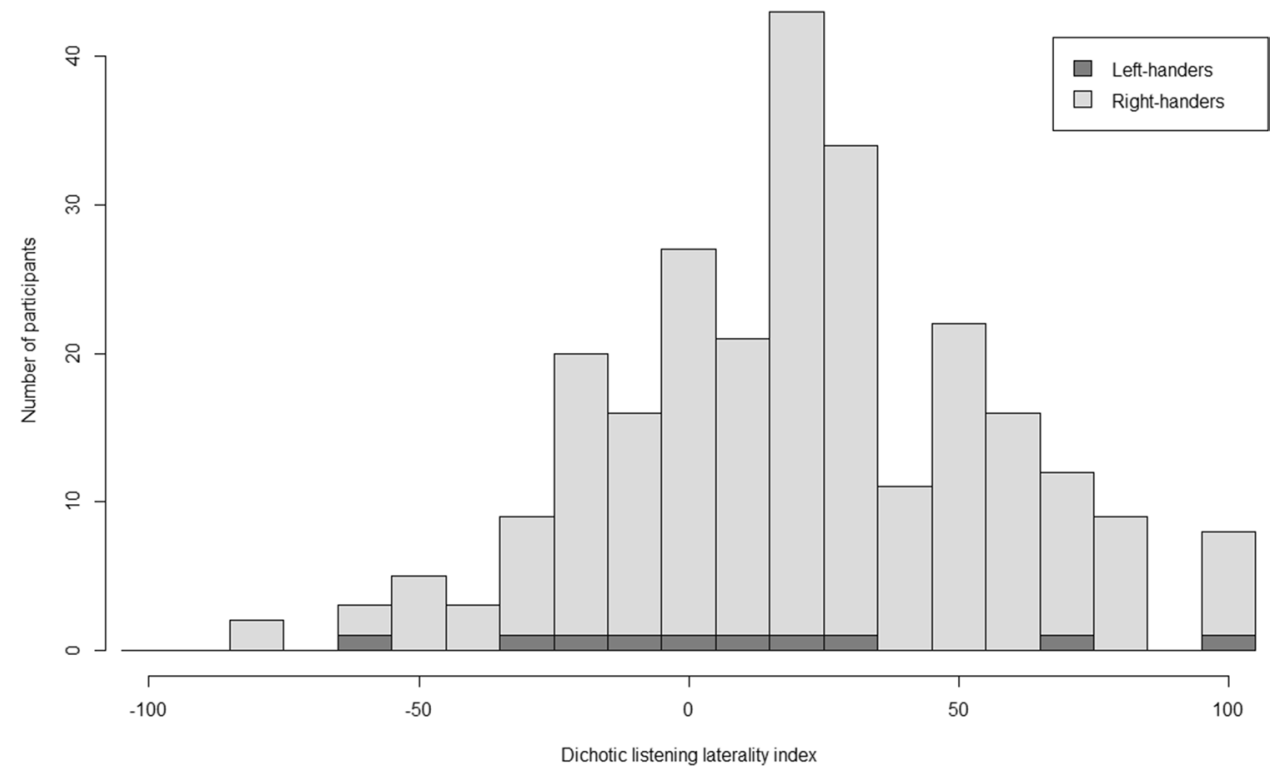

was positively correlated with the dichotic listening laterality index. More details of the model at the peak voxel are in Supplementary Table 2. In post hoc analysis of the corresponding unilateral grey matter volumes, smaller leftsided volume in this cluster was correlated with increasingly atypical left-ear advantage, with no change on the right side (Fig. 3c)(left $r=0.16, p=0.00826$; right $r=-0.08$, $p=0.157)$. There were no clusters identified with uncorrected $p<0.10$ within the Heschl's gyrus or planum temporale regions of interest of the auditory cortex.

When excluding the 10 left-handers, the brain-wide analysis resulted in a very similar statistical map to that from the main analysis $(r=0.91, p<1 \mathrm{e}-20)$. The peak associations were slightly weakened, as might be expected due to slightly decreased statistical power (Amygdala: $Z=3.27$; cerebellum: $Z=3.77$; pSTG: $Z=1.92$ ).

\section{Discussion}

We performed the largest study to date of brain structural asymmetry in relation to auditory processing of spoken syllables. The dataset that we used was roughly one order of magnitude larger than previous studies that attempted to link dichotic listening laterality to variations in brain structure (see "Introduction"). In general, relations between structural and functional variability of the brain are subtle and complex (Chen and Omiya 2014; Tzourio-Mazoyer et al. 2018; Batista-Garcia-Ramo and Fernandez-Verdecia 2018), and therefore studies of these relations are likely to benefit from larger samples than have often been used in the field.

In brain-wide voxel-wise asymmetry analysis, we found that two clusters, in the amygdala and cerebellum lobule
VI, were significantly associated with the dichotic listening laterality index. These findings are striking insofar as they implicate regions located outside of the cerebral cortex, which is often considered as the primary seat of lateralized cognition in the human brain (Ji et al. 2019). As these are both original findings in our dataset, we remain cautious and suggest that further replication studies are needed. Nonetheless, there are other indications in the literature of the potential relevance of these structures to language-related cognition:

As regards the amygdala, a previous fMRI study based on 13 individuals, in which target syllable detection was tested during dichotic listening, found that the left amygdala (among other regions) responded differently to left- and right-ear target detection (Pollmann et al. 2004). This suggested involvement of the left amygdala in right-ear advantage, and that the amygdala may be part of a broader circuit involved in auditory stimulus detection based on ambivalent sensory information (Pollmann et al. 2004). Other data have also suggested involvement of the amygdala in languagerelated networks. Stereo-electroencephalography (involving intracranial stimulation in patients with drug-resistant epilepsy) has shown functional connectivity of the amygdala with frontal and temporal regions important for language (Physiological Structural-Effective Connectivity Atlas: http://epi.fizica.unibuc.ro/atlas/). A recent fMRI study found the amygdala to be among regions showing left-lateralized activation during multi-modal, sentence-level task performance (Labache et al. 2019). Another recent fMRI study attempted to map relationships between subcortical structures and cortical networks, and found that the amygdala exhibits functional connectivity with the language network (Ji et al. 2019). This finding was flagged by the authors as 
A

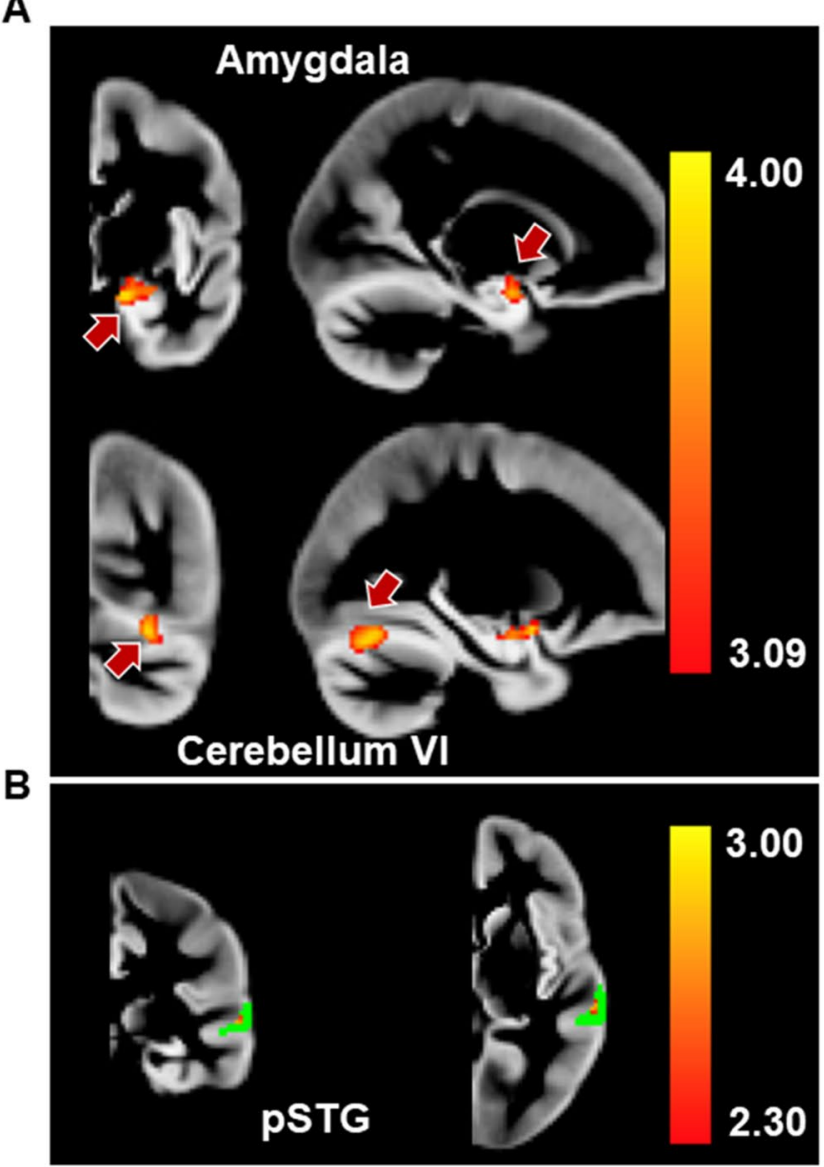

Fig. 3 a Two significant clusters associated with the dichotic listening laterality index, in voxel-wise grey matter asymmetry analysis; one in the amygdala, the other in cerebellum lobule VI. Both associations were in a positive direction (red-yellow indicates the association $Z$ score). b Region-of-interest analysis of voxel-wise grey matter volume asymmetry in relation to the dichotic listening laterality index. The posterior superior temporal region of interest is shown in green (voxels with at least $50 \%$ probability of belonging to the region according to the Harvard-Oxford atlas), and a tentative cluster showing positive association is mapped in red-yellow (indicating the asso-

unexpected and important to follow up in future research (Ji et al. 2019), as it does not currently have a clear or direct mechanistic interpretation. The amygdala is better known to be involved in emotion and motivation (Janak and Tye 2015).

Relatively more is known about the cerebellum in language-related processing (Desmond and Fiez 1998). The cerebellum has reciprocal connections with brain regions involved in phonological processing, i.e. the inferior frontal gyrus and lateral temporal cortex (Booth et al. 2007). We found a significant association between dichotic listening laterality and grey matter asymmetry specifically in cerebellum lobule VI, which was driven by a right-hemisphere increase of volume with atypical left-ear advantage.
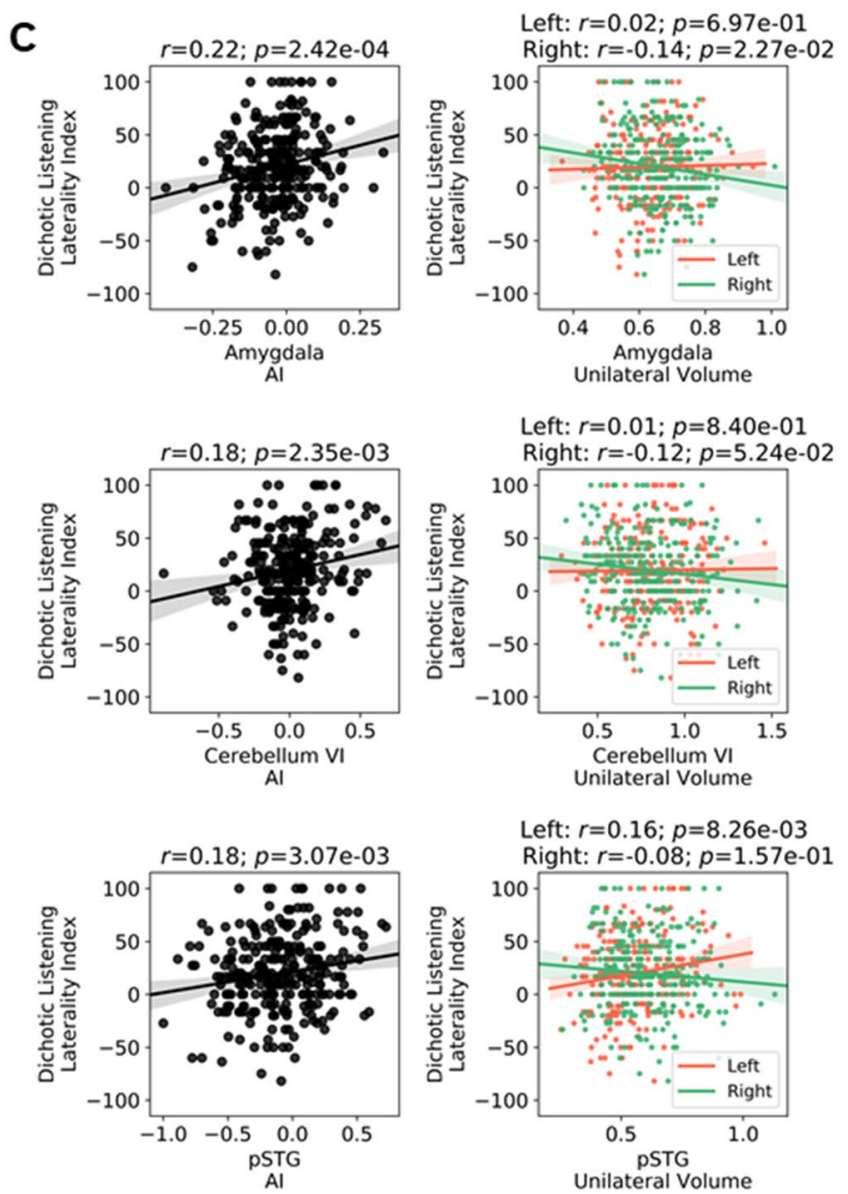

ciation $Z$ score). $\mathbf{c}$ Correlations between the dichotic listening laterality index and peak coordinate measures of grey matter volume, for the amygdala cluster (top), the cerebellum lobule VI cluster (middle), and the posterior superior temporal gyrus (pSTG) cluster (bottom). For each peak coordinate, correlations for voxel-wise grey matter asymmetry indexes (AI) as well as the corresponding unilateral left and right volume data are shown. Note that the primary analysis of AIs was based on General Linear Modelling including confound variables (see "Methods" section), but that correlations at the peak voxels are shown here for visualization

Language-related activation in the right cerebellum VI has been reported in language-task fMRI analysis (Stoodley and Schmahmann 2009) and is consistent with contralateral projections between the cerebellum and left-lateralized language network in the cerebral cortex (Jansen et al. 2005). Our data suggest that this reciprocal cerebellar-cortical functional laterality is linked to grey matter structural laterality in the cerebellum more noticeably than in the cerebral cortex and point to a contralateral role of the cerebellum in syllabic auditory processing. Another recent study, of two individuals each scanned across 31 MRI sessions, also showed functional connectivity of right cerebellum VI with the left cortical language network (Xue et al. 2020). Although tightly folded, the human cerebellar cortex was recently reported 
to have a surface area equal to roughly $4 / 5$ of the neocortex, whereas in the macaque the cerebellar cortex is equal in surface area to only around $1 / 3$ of the neocortex (Sereno et al. 2020). This suggests that the cerebellum has played an important role in the evolution of human cognition and behaviour, and language may be one adaptation to which the cerebellum contributes.

Developmentally, left-right differences of gene expression have been reported in the spinal cords and hindbrains of human embryos and fetuses, which may precede asymmetrical development of other brain regions (de Kovel et al. 2017). As a hindbrain structure, the cerebellum may therefore be a developmental source of laterality that affects other regions such as the cerebral cortex later on via asymmetrical, long-range connections. More research is required on hindbrain and subcortical roles in lateralized development and functioning of the human brain.

As regards auditory cortex, when using a relatively loose voxel-level $Z$ threshold, we found a trend of association between the dichotic listening laterality index and grey matter asymmetry within the posterior superior temporal cortex, located next to Heschl's gyrus and the planum temporale, where smaller left-sided volume was associated with increasingly atypical left-ear advantage. This direction of effect suggests that left-hemisphere dominance for auditory speech processing is favoured by greater left-hemisphere grey matter volume in a restricted region of auditory cortex. In a previous study contrasting 34 participants with left hemisphere language dominance and 21 with right hemisphere dominance (Greve et al. 2013), analysis using surface-based registration across hemispheres and subjects found a small but significant cluster in the superior temporal gyrus, which overlapped with the planum temporale. The peak location reported in their paper (Greve et al. 2013) does not match the cluster in our analysis, although the full extent of their cluster might have overlapped with ours (this is difficult to judge due to the different methods used). Our finding lends support to the existence of an anatomical correlate of functional language dominance within auditory cortex. However, functional laterality for auditory processing of spoken syllables cannot necessarily be taken to reflect broader hemispheric language dominance (Tzourio-Mazoyer et al. 2018). Regardless, our finding is consistent with previous reports insofar as any superior temporal structure-function link is likely to be subtle, and not substantially predictive.

A previous fMRI study using dichotic listening in 104 participants found leftward asymmetries of activation in the posterior superior temporal gyrus, located adjacent to symmetrically activated areas (Westerhausen et al. 2014). Again, while the peak location of their asymmetrical cluster in the superior temporal gyrus did not co-locate with our anatomical cluster, it is possible that their broader cluster overlaps with ours, although it is not possible to be certain with the information given in the paper. We have deposited our full mapping results from the dichotic-asymmetry-VBM analysis in the Neurovault database (https://identifiers.org/ neurovault.collection:8231), in order to facilitate comparisons with future studies.

It is unclear how hemispheric regional grey matter volume differences might relate to functionally relevant differences of neuronal circuitry for lateralized functions. As mentioned in the "Introduction", previous studies based on post mortem analysis or neurite orientation dispersion and density imaging have indicated hemispheric differences of cortical grey matter microstructure, especially for superior temporal regions involved in auditory processing (Ocklenburg et al. 2018; Schmitz et al. 2019). One of these studies also linked faster neurophysiological processing of speech sounds in the left planum temporale (as measured by electroencephalography) to a higher inferred density of neurites (Ocklenburg et al. 2018). However, the neurophysiological measure did not correlate with overall planum temporale grey matter volume in that study, which underscores that there is no direct link from grey matter macrostructural variability to functional variability. Nonetheless, findings such as ours, which link specific regional grey matter volumes to functional variability, provide targets for further investigation using more high resolution structural and functional techniques.

A limitation of our study was the time that elapsed between MRI scanning and dichotic listening, which ranged from 1.6 months to 15.3 years, and averaged 5.8 years. A necessary assumption of our study was therefore relative stability of brain structural and functional asymmetry over periods of this length in healthy adulthood, in a dataset of predominantly young adults. To partly address this potential issue, in addition to the age at scanning, we included the time difference between scanning and dichotic listening as a confound variable in our general linear modelling. Previous studies have indicated that age has significant but small effects on brain structural asymmetry (Fjell et al. 2015; Kong et al. 2018), such that age-related changes are likely to have limited impact during e.g. one decade of healthy adult life. Nonetheless, the statistical power to detect structure-function links in our study may have been reduced by this aspect.

Other limitations may relate to our Dutch-language, remote version of the dichotic listening task. Firstly, there was a substantial loss of data from 643 participants who attempted the task, to a final number of 293 with usable data (a further 12 were excluded due to quality control of MRI data). The loss of dichotic listening data occurred due to a combination of factors: failure to answer the setup questions correctly on left-right placement and balance of the headphones (the task was performed remotely at home), and high error rates which also incorporated missing responses. 
In principle, it is possible that loss of data was non-random, and that this led to biases in the dataset. However, there is no obvious reason to expect that brain asymmetrical structure-function links would be selectively sampled among those who provided usable data.

We also found that performance was strongly confounded with VOT (i.e. voice onset time) category, in the contrasts between short and long VOT stimuli, and we therefore excluded these pairs. This left twelve syllable pairs for calculating the dichotic listening laterality index, which may have contributed to error variance as the index was based on relatively few datapoints per participant. A previous study of the effects of VOT category in dichotic listening found the opposite pattern, i.e. long VOT stimuli were perceived dominantly, in contrasts with short VOT stimuli (Rimol et al. 2006). Our short VOT stimuli had longer overall durations than our long VOT stimuli (see "Methods" section), and started before the long VOT stimuli, as all of the stimuli were aligned at the release of the stop consonant. These factors may have favoured dominant perception of short over long VOT stimuli in our study, regardless of the ear of presentation. To our knowledge, ours was the first Dutch implementation of consonant-vowel-syllable dichotic listening, and it has previously been found that performance on this task varies across languages (Bless et al. 2015a). More general limitations of dichotic listening for assessing hemispheric dominance for speech processing have been discussed extensively before (Hugdahl 2011; Westerhausen 2019).

As the MRI data in the BIG dataset came from multiple separate, smaller-scale MRI studies that were performed over a period of roughly fifteen years, there was substantial heterogeneity of scanners and scanning protocols (see "Methods" section and Supplementary Table 1). We included 'scanner' as a confound variable in our general linear model analysis, which also adjusted for differences of field strength and other consistent properties of individual scanners. It is also likely that applying the denominator of the asymmetry index $(L-R) /((L+R) 2))$ reduced the influence of scanning heterogeneity factors that impacted the measurement of both hemispheres equally. Nonetheless, if similar-sized studies could be carried out in the future using single scanners and scanning protocols, then reduced heterogeneity might improve the potential for detecting structure-function links. However, a doubt then arises about whether such findings would generalize across different scanners and protocols.

Rather than VBM, which measures grey matter volumes, future studies of brain structural relations to dichotic listening laterality may benefit from cortical surface-based approaches that separately measure surface area and thickness as distinct variables (Desikan et al. 2006). Improved mapping of asymmetry in this context may be achieved through hemispheric co-registration (Maingault et al. 2016; Greve et al. 2013). The precise mapping of VBM clusters is also an issue. For example, the cerebellar cluster identified in the present study overlapped at its upper edge with the nearby occipital fusiform gyrus, such that there may have been a degree of cortical contribution to this effect, but the resolution of the method was not sufficient to judge this with certainty (Fig. 3). Other aspects of brain structure such as white matter tracts should also be investigated in larger datasets than used previously, through imaging modalities such as diffusion tensor imaging.

In our study, only 10 of the 281 participants with dichotic listening and brain MRI data were left-handed, due to lefthandedness having been an exclusion criterion in some of the studies from which the BIG dataset was compiled (Guadalupe et al. 2014). There is an elevated rate of right-hemisphere language dominance among left-handers compared to right-handers (Mazoyer et al. 2014; Knecht et al. 2000; Van der Haegen and Brysbaert 2018), such that one of our ten left-handers would be expected to have right-hemisphere language dominance (Mazoyer et al. 2014). However, as the number of left-handers was too low for reliable statistical analysis, and we saw no association of the dichotic listening laterality index with handedness, we did not consider handedness further in our analysis. Handedness has also been found to be of limited relevance to cerebral cortical macrostructural brain asymmetry measures (Guadalupe et al. 2014; Kong et al. 2018). Larger studies, or studies which deliberately select for left-handed people, will be required to tease out potential relations between handedness, brain structure and auditory processing assessed by dichotic listening.

In summary, we have used MRI and dichotic listening data from 281 healthy subjects to contribute to mapping brain asymmetrical structure-function links, specifically with regard to grey matter and auditory processing of spoken syllables. The results identified novel associations involving the amygdala and cerebellum lobule VI, as well as a tentative cluster within auditory cortex. These findings motivate future studies of brain structural and language-related functional laterality that consider subcortical and hindbrain structures, in addition to the cerebral cortex.

Author contributions TG: study conception, dichotic listening test design, data preparation, drafting of manuscript. X-ZK: study conception, brain image analysis, drafting of manuscript. SEAA: data preparation, editing of manuscript. SEF: study conception, editing of manuscript. CF: study conception and direction, drafting and editing of manuscript.

Funding Open Access funding enabled and organized by Projekt DEAL. This research was funded by the Max Planck Society (Germany). This work made use of the BIG (Brain Imaging Genetics) database, first established in Nijmegen, The Netherlands, in 2007. This resource is now part of Cognomics (www.cognomics.nl), a joint 
initiative by researchers of the Donders Centre for Cognitive Neuroimaging, the Human Genetics and Cognitive Neuroscience departments of the Radboud University Medical Center (Radboudumc), and the Max Planck Institute for Psycholinguistics in Nijmegen.

Availability of data and material Raw behavioural and imaging data were obtained from the BIG database and are available with the permission of the Cognomics Initiative (http://www.cognomics.nl/). Restrictions apply related to the privacy and consent of the research participants. Statistical maps from the brain imaging analyses, before thresholding, have been uploaded to NeuroVault (https://identifiers.org/ neurovault.collection:8231).

Code availability Code for the brain imaging analyses is available in GitHub (https://github.com/Conxz/dichotic_vbm).

\section{Compliance with ethical standards}

Conflict of interest The authors report no potential conflicts of interest regarding this study.

Ethics approval Ethical approval was from the 'Commissie Mensgebonden Onderzoek' (Committee on Research Involving Human Subjects) of the region Arnhem-Nijmegen.

Consent to participate All participants gave written, informed consent to participate.

Open Access This article is licensed under a Creative Commons Attribution 4.0 International License, which permits use, sharing, adaptation, distribution and reproduction in any medium or format, as long as you give appropriate credit to the original author(s) and the source, provide a link to the Creative Commons licence, and indicate if changes were made. The images or other third party material in this article are included in the article's Creative Commons licence, unless indicated otherwise in a credit line to the material. If material is not included in the article's Creative Commons licence and your intended use is not permitted by statutory regulation or exceeds the permitted use, you will need to obtain permission directly from the copyright holder. To view a copy of this licence, visit http://creativecommons.org/licenses/by/4.0/.

\section{References}

Andersson J, Jenkinson M, Smith S (2010) Non-linear registration, aka Spatial normalisation. FMRIB technical report TR07JA2.

Batista-Garcia-Ramo K, Fernandez-Verdecia CI (2018) What we know about the brain structure-function relationship. Behav Sci (Basel). https://doi.org/10.3390/bs8040039

Bless JJ, Westerhausen R, Arciuli J, Kompus K, Gudmundsen M, Hugdahl K (2013) "Right on all Occasions?" - on the feasibility of laterality research using a smartphone dichotic listening application. Front Psychol 4:42. https://doi.org/10.3389/fpsyg.2013.00042

Bless JJ, Westerhausen R, Torkildsenv JvL, Gudmundsen M, Kompus K, Hugdahl K (2015a) Laterality across languages: results from a global dichotic listening study using a smartphone application. Lateral Asymmetries Body Brain Cogn. https://doi. org/10.1080/1357650x.2014.997245

Bless JJ, Westerhausen R, von Koss TJ, Gudmundsen M, Kompus K, Hugdahl K (2015b) Laterality across languages: results from a global dichotic listening study using a smartphone application.
Laterality 20(4):434-452. https://doi.org/10.1080/13576 50x.2014.997245

Booth JR, Wood L, Lu D, Houk JC, Bitan T (2007) The role of the basal ganglia and cerebellum in language processing. Brain Res 1133(1):136-144. https://doi.org/10.1016/j.brainres.2006.11.074

Chance SA (2014) The cortical microstructural basis of lateralized cognition: a review. Front Psychol 5:820. https://doi.org/10.3389/ fpsyg.2014.00820

Chen C, Omiya Y (2014) Brain asymmetry in cortical thickness is correlated with cognitive function. Front Hum Neurosci 8:877. https ://doi.org/10.3389/fnhum.2014.00877

Collinson SL, Mackay CE, Jiaqing O, James AC, Crow TJ (2009) Dichotic listening impairments in early onset schizophrenia are associated with reduced left temporal lobe volume. Schizophr Res 112(1-3):24-31. https://doi.org/10.1016/j.schres.2009.03.034

de Kovel CGF, Francks C (2019) The molecular genetics of hand preference revisited. Sci Rep 9(1):5986. https://doi.org/10.1038/ s41598-019-42515-0

de Kovel CGF, Lisgo S, Karlebach G, Ju J, Cheng G, Fisher SE, Francks C (2017) Left-right asymmetry of maturation rates in human embryonic neural development. Biol Psychiat 82(3):204-212. https://doi.org/10.1016/j.biopsych.2017.01.016

de Kovel CGF, Lisgo SN, Fisher SE, Francks C (2018) Subtle left-right asymmetry of gene expression profiles in embryonic and foetal human brains. Sci Rep 8(1):12606. https://doi. org/10.1038/s41598-018-29496-2

de Kovel CGF, Carrion-Castillo A, Francks C (2019a) A large-scale population study of early life factors influencing left-handedness. Sci Rep 9(1):584. https://doi.org/10.1038/s41598-01837423-8

de Kovel CGF, Carrión-Castillo A, Francks C (2019b) A large-scale population study of early life factors influencing left-handedness. Sci Rep 9(1):584. https://doi.org/10.1038/s41598-018-37423-8

Desikan RS, Segonne F, Fischl B, Quinn BT, Dickerson BC, Blacker D (2006) An automated labeling system for subdividing the human cerebral cortex on MRI scans into gyral based regions of interest. Neuroimage 31:968

Desmond JE, Fiez JA (1998) Neuroimaging studies of the cerebellum: language, learning and memory. Trends Cogn Sci 2(9):355-362. https://doi.org/10.1016/s1364-6613(98)01211-x

Diedrichsen J, Balsters JH, Flavell J, Cussans E, Ramnani N (2009) A probabilistic MR atlas of the human cerebellum. Neuroimage 46(1):39-46. https://doi.org/10.1016/j.neuroimage.2009.01.045

Douaud G, Smith S, Jenkinson M, Behrens T, Johansen-Berg H, Vickers J, James S, Voets N, Watkins K, Matthews PM, James A (2007) Anatomically related grey and white matter abnormalities in adolescent-onset schizophrenia. Brain 130(9):2375-2386. https ://doi.org/10.1093/brain/awm184

Eyler LT, Vuoksimaa E, Panizzon MS, Fennema-Notestine C, Neale MC, Chen C-H, Jak A, Franz CE, Lyons MJ, Thompson WK, Spoon KM, Fischl B, Dale AM, Kremen WS (2013) Conceptual and data-based investigation of genetic influences and brain asymmetry: a twin study of multiple structural phenotypes. J Cogn Neurosci 26(5):1100-1117. https://doi.org/10.1162/jocn_a_00531

Fjell AM, Grydeland H, Krogsrud SK, Amlien I (2015) Development and aging of cortical thickness correspond to genetic organization patterns. Proc Natl Sci USA 112(50):15462-15467. https://doi. org/10.1073/pnas.1508831112

Francks C (2015) Exploring human brain lateralization with molecular genetics and genomics. Ann N Y Acad Sci 1359(1):1-13. https:// doi.org/10.1111/nyas. 12770

Franke B, Vasquez AA, Veltman JA, Brunner HG, Rijpkema M, Fernandez G (2010) Genetic variation in CACNA1C, a gene associated with bipolar disorder, influences brainstem rather than gray matter volume in healthy individuals. Biol Psychiat 68(6):586588. https://doi.org/10.1016/j.biopsych.2010.05.037 
Frazier JA, Chiu S, Breeze JL, Makris N, Lange N, Kennedy DN, Herbert MR, Bent EK, Koneru VK, Dieterich ME, Hodge SM, Rauch SL, Grant PE, Cohen BM, Seidman LJ, Caviness VS, Biederman J (2005) Structural brain magnetic resonance imaging of limbic and thalamic volumes in pediatric bipolar disorder. Am J Psychiatry 162(7):1256-1265. https://doi.org/10.1176/appi.ajp.162.7.1256

Geschwind N, Levitsky W (1968) Human brain: left-right asymmetries in temporal speech region. Science 161(3837):186-187

Goldstein JM, Goodman JM, Seidman LJ, Kennedy DN, Makris N, Lee H, Tourville J, Caviness VS Jr, Faraone SV, Tsuang MT (1999) Cortical abnormalities in schizophrenia identified by structural magnetic resonance imaging. Arch Gen Psychiatry 56(6):537547. https://doi.org/10.1001/archpsyc.56.6.537

Good CD, Johnsrude IS, Ashburner J, Henson RNA, Friston KJ, Frackowiak RSJ (2001) A voxel-based morphometric study of ageing in 465 normal adult human brains. NeuroImage 14(1):21-36. https ://doi.org/10.1006/nimg.2001.0786

Greve DN, Van der Haegen L, Cai Q, Stufflebeam S, Sabuncu MR, Fischl B, Brysbaert M (2013) A surface-based analysis of language lateralization and cortical asymmetry. J Cogn Neurosci 25(9):1477-1492. https://doi.org/10.1162/jocn_a_00405

Guadalupe T, Willems RM, Zwiers MP, Arias Vasquez A, Hoogman M, Hagoort P, Fernandez G, Buitelaar J, Franke B, Fisher SE, Francks C (2014) Differences in cerebral cortical anatomy of leftand right-handers. Front Psychol 5:261. https://doi.org/10.3389/ fpsyg.2014.00261

Hugdahl K (2011) Fifty years of dichotic listening research-still going and going and. Brain Cogn 76(2):211-213. https://doi. org/10.1016/j.bandc.2011.03.006

Hugdahl K, Westerhausen R (2016) Speech processing asymmetry revealed by dichotic listening and functional brain imaging. Neuropsychologia 93(Pt B):466-481. https://doi.org/10.1016/j.neuro psychologia.2015.12.011

Hutsler JJ (2003) The specialized structure of human language cortex: pyramidal cell size asymmetries within auditory and language-associated regions of the temporal lobes. Brain Lang 86(2):226-242

Hutsler J, Galuske RA (2003) Hemispheric asymmetries in cerebral cortical networks. Trends Neurosci 26(8):429-435

Janak PH, Tye KM (2015) From circuits to behaviour in the amygdala. Nature 517(7534):284-292. https://doi.org/10.1038/nature14188

Jansen A, Flöel A, Van Randenborgh J, Konrad C, Rotte M, Förster AF, Deppe M, Knecht S (2005) Crossed cerebro-cerebellar language dominance. Hum Brain Mapp 24(3):165-172. https://doi. org/10.1002/hbm.20077

Jansen A, Liuzzi G, Deppe M, Kanowski M, Ölschläger C, Albers JM, Schlaug G, Knecht S (2010) Structural correlates of functional language dominance: a voxel-based morphometry study. J Neuroimaging 20(2):148-156. https://doi.org/10.111 1/j.1552-6569.2009.00367.x

Ji JL, Spronk M, Kulkarni K, Repovš G, Anticevic A, Cole MW (2019) Mapping the human brain's cortical-subcortical functional network organization. Neuroimage 185:35-57. https://doi. org/10.1016/j.neuroimage.2018.10.006

Josse G, Kherif F, Flandin G, Seghier ML, Price CJ (2009) Predicting language lateralization from gray matter. J Neurosci 29(43):13516-13523. https://doi.org/10.1523/jneur osci.1680-09.2009

Kasprian G, Langs G, Brugger PC, Bittner M, Weber M, Arantes M, Prayer D (2011) The prenatal origin of hemispheric asymmetry: an in utero neuroimaging study. Cereb Cortex 21(5):1076-1083. https://doi.org/10.1093/cercor/bhq179

Keller SS, Roberts N, Baker G, Sluming V, Cezayirli E, Mayes A, Eldridge P, Marson AG, Wieshmann UC (2018) A voxel-based asymmetry study of the relationship between hemispheric asymmetry and language dominance in Wada tested patients. Hum Brain Mapp 39(7):3032-3045. https://doi.org/10.1002/hbm.24058

Knecht S, Drager B, Deppe M, Bobe L, Lohmann H, Floel A, Ringelstein EB, Henningsen H (2000) Handedness and hemispheric language dominance in healthy humans. Brain 123(Pt 12):2512-2518

Kong XZ, Mathias SR, Guadalupe T, Glahn DC, Franke B (2018) Mapping cortical brain asymmetry in 17,141 healthy individuals worldwide via the ENIGMA Consortium. Proc Natl Acad Sci 115(22):E5154-e5163. https://doi.org/10.1073/pnas.1718418115

Kong X-Z, Postema MC, Guadalupe T, de Kovel C, Boedhoe PSW, Hoogman M, Mathias SR, van Rooij D, Schijven D, Glahn DC, Medland SE, Jahanshad N, Thomopoulos SI, Turner JA, Buitelaar J, van Erp TGM, Franke B, Fisher SE, van den Heuvel OA, Schmaal L, Thompson PM (2020) Francks C Mapping brain asymmetry in health and disease through the ENIGMA consortium. Hum Brain Mapp. https://doi.org/10.1002/hbm.25033

Labache L, Joliot M, Saracco J, Jobard G, Hesling I, Zago L, Mellet E, Petit L, Crivello F, Mazoyer B, Tzourio-Mazoyer N (2019) A SENtence Supramodal Areas AtlaS (SENSAAS) based on multiple task-induced activation mapping and graph analysis of intrinsic connectivity in 144 healthy right-handers. Brain Struct Funct 224(2):859-882. https://doi.org/10.1007/s0042 9-018-1810-2

Maingault S, Tzourio-Mazoyer N, Mazoyer B, Crivello F (2016) Regional correlations between cortical thickness and surface area asymmetries: a surface-based morphometry study of 250 adults. Neuropsychologia 93(Pt B):350-364. https://doi.org/10.1016/j. neuropsychologia.2016.03.025

Mazoyer B, Zago L, Jobard G, Crivello F, Joliot M, Perchey G, Mellet E, Petit L, Tzourio-Mazoyer N (2014) Gaussian mixture modeling of hemispheric lateralization for language in a large sample of healthy individuals balanced for handedness. PLOS ONE 9(6):e101165. https://doi.org/10.1371/journal.pone.0101165

McManus IC (2009) The history and geography of human handedness. In: Sommer IEC, Kahn RS (eds) Language lateralization and psychosis. Cambridge University Press, Cambridge, pp 37-58. https ://doi.org/10.1017/CBO9780511576744.004

McManus IC, Davison A, Armour JA (2013) Multilocus genetic models of handedness closely resemble single-locus models in explaining family data and are compatible with genome-wide association studies. Ann N Y Acad Sci 1288:48-58. https://doi. org/10.1111/nyas.12102

Moerel M, De Martino F, Formisano E (2014) An anatomical and functional topography of human auditory cortical areas. Front Neurosc. https://doi.org/10.3389/fnins.2014.00225

Munafo MR, Flint J (2010) How reliable are scientific studies? Br J Psychiatry 197(4):257-258. https://doi.org/10.1192/bjp. bp. 109.069849

Nygard M, Loberg EM, Craven AR, Ersland L, Berle JO, Kroken RA, Johnsen E, Hugdahl K (2013) Dichotic listening, executive functions and grey matter cortical volume in patients with schizophrenia and healthy controls. Scand J Psychol 54(6):443-450. https:// doi.org/10.1111/sjop. 12080

Ocklenburg S, Schlaffke L, Hugdahl K, Westerhausen R (2014) From structure to function in the lateralized brain: how structural properties of the arcuate and uncinate fasciculus are associated with dichotic listening performance. Neurosci Lett 580:32-36. https:// doi.org/10.1016/j.neulet.2014.07.044

Ocklenburg S, Schmitz J, Moinfar Z, Moser D, Klose R, Lor S, Kunz G, Tegenthoff M, Faustmann P, Francks C, Epplen JT, Kumsta R, Güntürkün O (2017) Epigenetic regulation of lateralized fetal spinal gene expression underlies hemispheric asymmetries. eLife 6:e22784. https://doi.org/10.7554/eLife.22784

Ocklenburg S, Friedrich P, Fraenz C, Schluter C, Beste C, Gunturkun O, Genc E (2018) Neurite architecture of the planum temporale 
predicts neurophysiological processing of auditory speech. Sci Adv 4(7):eaar6830. https://doi.org/10.1126/sciadv.aar6830

Papadatou-Pastou M, Ntolka E, Schmitz J, Martin M, Munafò MR, Ocklenburg S, Paracchini S (2020) Human handedness: a metaanalysis. Psychol Bull. https://doi.org/10.1037/bul0000229

Pollmann S, Lepsien J, Hugdahl K, von Cramon DY (2004) Auditory target detection in dichotic listening involves the orbitofrontal and hippocampal paralimbic belts. Cereb Cortex 14(8):903-913. https ://doi.org/10.1093/cercor/bhh049

Postema MC, Carrion-Castillo A, Fisher SE, Vingerhoets G, Francks C (2020) The genetics of situs inversus without primary ciliary dyskinesia. Sci Rep Uk. https://doi.org/10.1038/s41598-020-60589-z

Rimol LM, Eichele T, Hugdahl K (2006) The effect of voice-onset-time on dichotic listening with consonant-vowel syllables. Neuropsychologia 44(2):191-196. https://doi.org/10.1016/j.neuropsych ologia.2005.05.006

Schmitz J, Fraenz C, Schluter C, Friedrich P, Jung RE, Gunturkun O, Genc E, Ocklenburg S (2019) Hemispheric asymmetries in cortical gray matter microstructure identified by neurite orientation dispersion and density imaging. Neuroimage. https://doi. org/10.1016/j.neuroimage.2019.01.079

Sereno MI, Diedrichsen J, Tachrount M, Testa-Silva G, d'Arceuil H, De Zeeuw C (2020) The human cerebellum has almost $80 \%$ of the surface area of the neocortex. Proc Natl Acad Sci. https://doi. org/10.1073/pnas.2002896117

Smith SM, Jenkinson M, Woolrich MW, Beckmann CF, Behrens TEJ, Johansen-Berg H, Bannister PR, De Luca M, Drobnjak I, Flitney DE, Niazy RK, Saunders J, Vickers J, Zhang Y, De Stefano N, Brady JM, Matthews PM (2004) Advances in functional and structural MR image analysis and implementation as FSL. NeuroImage 23:S208-S219. https://doi.org/10.1016/j.neuroimage.2004.07.051

Stoodley CJ, Schmahmann JD (2009) Functional topography in the human cerebellum: a meta-analysis of neuroimaging studies. Neuroimage 44(2):489-501. https://doi.org/10.1016/j.neuroimage .2008 .08 .039
Toga AW, Thompson PM (2003) Mapping brain asymmetry. Nat Rev Neurosci 4(1):37-48

Tzourio-Mazoyer N, Seghier ML (2016) The neural bases of hemispheric specialization. Neuropsychologia 93:319-324. https://doi. org/10.1016/j.neuropsychologia.2016.10.010

Tzourio-Mazoyer N, Crivello F, Mazoyer B (2018) Is the planum temporale surface area a marker of hemispheric or regional language lateralization? Brain Struct Funct 223(3):1217-1228. https://doi. org/10.1007/s00429-017-1551-7

Van der Haegen L, Brysbaert M (2018) The relationship between behavioral language laterality, face laterality and language performance in left-handers. PLoS ONE 13(12):e0208696. https:// doi.org/10.1371/journal.pone.0208696

Westerhausen R (2019) A primer on dichotic listening as a paradigm for the assessment of hemispheric asymmetry. Laterality 24(6):740-771. https://doi.org/10.1080/1357650X.2019.1598426

Westerhausen R, Kompus K, Hugdahl K (2014) Mapping hemispheric symmetries, relative asymmetries, and absolute asymmetries underlying the auditory laterality effect. Neuroimage 84:962-970. https://doi.org/10.1016/j.neuroimage.2013.09.074

Xue A, Kong R, Yang Q, Eldaief MC, Angeli P, DiNicola LM, Braga RM, Buckner RL, Yeo BTT (2020) The detailed organization of the human cerebellum estimated by intrinsic functional connectivity within the individual. bioRxiv. https://doi. org/10.1101/2020.09.15.297911

Zago L, Herve PY, Genuer R, Laurent A, Mazoyer B, Tzourio-Mazoyer N, Joliot M (2017) Predicting hemispheric dominance for language production in healthy individuals using support vector machine. Hum Brain Mapp. https://doi.org/10.1002/hbm.23770

Publisher's Note Springer Nature remains neutral with regard to jurisdictional claims in published maps and institutional affiliations. 